\title{
Human Brown Adipose Tissue Plasticity: Hormonal and Environmental Manipulation
}

\author{
Francesco S. Celi
}

\begin{abstract}
Brown adipose tissue (BAT), brown-in-white ("brite") and "beige" adipocytes share the unique ability of converting chemical energy into heat and play a critical role in the adaptive thermogenesis response promoting nonshivering thermogenesis. Uncoupling Protein-1 (UCP1), which allows the uncoupling of substrate oxidation from phosphorylation of ADP, represents the molecular signature of BAT and beige adipocytes. Until recently, the physiologic role of BAT and beige adipocytes depots was thought to be limited to small mammalians and newborns.

The discovery of BAT in adult humans and the demonstration of the presence of inducible BAT activity in white adipose tissue by beige adipocytes have generated enthusiasm as potential targets for treatment of obesity and other disorders due to sustained positive energy balance. These findings are particularly important since in vitro studies have demonstrated that preadipocytes can be directed toward a common brown phenotype by multiple pathways that, in turn, may be exploited for therapeutic interventions. In adult humans, BAT activity is more evident in deep neck fat depots and, to a lesser degree, in subcutaneous adipose tissue, with a transcriptome signature resembling the rodent beige fat. This observation supports the hypothesis that human BAT activity and capacity can be modulated. To this end, we have directed our translational research program toward the characterization of beige fat in humans and on the effects of hormonal and environmental drivers in the adaptive thermogenesis response. Mild cold exposure, within the temperature range commonly employed in climate-controlled buildings, is sufficient to generate a significant increase in non-shivering thermogenesis driven by BAT and beige adipocyte activation. In turn, adaptive thermogenesis generates a specific hormonal signature and promotes glucose disposal. Chronic exposure to mild cold induces expansion of BAT mass and activity, whereas exposure to warm climate abrogates them. Additionally, the metabolic effects of BAT mass expansion are evident only upon stimulation of BAT activity, indicating that both
\end{abstract}

\section{F. S. Celi $(\square)$}

Division of Endocrinology Diabetes and Metabolism, Department of Internal Medicine,

Virginia Commonwealth University, Richmond, VA, USA

e-mail: Francesco.celi@vchuhealth.org

B. Spiegelman (ed.), Hormones, Metabolism and the Benefits of Exercise,

Research and Perspectives in Endocrine Interactions,

https://doi.org/10.1007/978-3-319-72790-5_1 
expansion and activation of BAT are necessary and complementary strategies to pursue. From an experimental standpoint, human preadipocytes represent a viable experimental platform to mechanistically interrogate different pathways that are able to expand and activate browning. Our laboratory has focused on studying FGF-21, and FNDC5/irisin in their capacity to promote the browning process. Compared to a white differentiation medium, the addition of either FGF-21 or FNDC5 results in a reprogramming toward a brown phenotype, as indicated by the display of brown transcriptome signature and, functionally, by the increase in oxygen consumption following catecholamine treatment, indicating an increase in substrate utilization. Collectively, the integration of a detailed assessment of human physiology with mechanistic observations in cell culture systems can provide a unique opportunity to translate observations from experimental models to actionable therapeutic targets.

\section{Introduction}

The unique ability of mammalians (and to some degree avians; Vianna et al. 2001) to maintain their core temperature independently of the environmental temperature has allowed their evolutionary success and, compared to poikilothermic species, to the wide distribution of species across climates. This response is defined as "adaptive thermogenesis," which describes the concerted physiologic responses aimed at defending the core temperature from low environmental temperatures, preserving the individual's ideal core temperature for biologic functions (Stocks et al. 2004). The main components of this response are thermal insulation, non-shivering thermogenesis, and shivering thermogenesis (Celi et al. 2015). From an evolutionary perspective, the excess of energy expenditure aimed at maintaining the core temperature represents a tradeoff between survival and maintenance of energy stores, since the availability of energy usually represents the limiting factor to growth and reproduction for organisms. To this end, moving from an insulative response, which is energy neutral, to a non-shivering and eventually shivering thermogenesis will require a progressively greater dissipation of energy stores (Fig. 1). Additionally, the relative size of the organism and the presence of fur dictate the relative importance of the insulative versus thermogenic response, with smaller organisms being biased toward the latter because of their high surface area-to-volume ratio, which, despite the presence of optimal insulation, will still promote thermal dispersion (Phillips and Heath 1995). Hence, modulation of energy expenditure plays a critical role in the maintenance of core temperature in small mammalians (Ravussin and Galgani 2011). Compared to humans, the capacity of adaptive thermogenesis in small rodents is significantly greater, resulting in an at least twofold increase in total energy expenditure, thus enabling survival in a cold environment, albeit at a price of a compensatory increase in food intake (Ravussin et al. 2014). Over the recent 


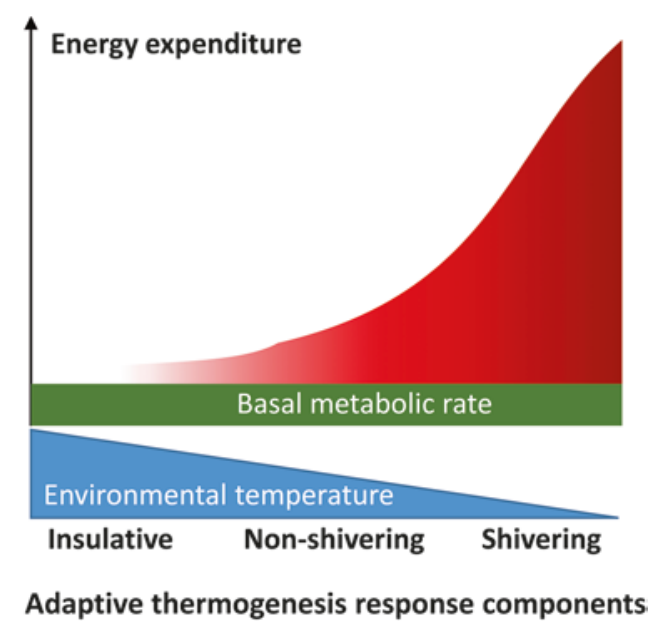

Fig. 1 Model of adaptive thermogenesis. As the environmental temperature decreases from thermoneutrality, the adaptive thermogenesis response moves from insulative to non-shivering and eventually shivering thermogenesis. This progression is mirrored by an increase in the energy expenditure required to maintain the core temperature. Green, energy expenditure due to basal metabolic rate; red, expenditure due to adaptive thermogenesis

evolutionary development of the species, humans have gained the ability to modulate their environment using garments and climate-controlled buildings. As a result, exposure to cold is a relatively unusual condition for them and, unless acclimated, individuals tend to respond by shivering thermogenesis, whereby heat is a side product of uncontrolled muscle fasciculation. Of interest, upon prolonged exposure to cold, individuals become resilient and do not display shivering thermogenesis (Davis 1961), indicating that other mechanisms are recruited.

\section{BAT and the Adaptive Thermogenesis Response}

BAT has the unique capability of converting energy stores into heat by virtue of the Uncoupling Protein 1 (UCP1), which promotes a proton leak in the inner membrane of the mitochondria, dissociating the oxidative phosphorylation of substrate from the generation of ATP. This process in essence shunts the chemical energy into heat, which is in turn dissipated in the circulation (Nedergaard et al. 2001). The rapid activation and inactivation of the UCP1-driven energy dissipation thus represents a valuable mechanism that is able to increase efficiently the metabolic rate on demand by non-shivering thermogenesis, ultimately promoting the survival of the organism against unfavorable environmental conditions with the least possible consumption of energy stores (Celi 2009). 
Aside from the anatomically defined intrascapular BAT, in mice non-shivering thermogenesis is generated in other adipose tissue depots, in particular inguinal, by "beige" or "brown-in-white (brite)" adipocytes (Ishibashi and Seale 2010; Petrovic et al. 2010), whose mass and activity can be modulated by various signal hormones and cytokines, showing a remarkable plasticity (Diaz et al. 2014).

\section{Implications of the Rediscovery of BAT in Adult Humans}

Several reports recognized the presence of brown adipocytes in adult humans, either as incidental findings (Huttunen et al. 1981) or as a nuisance in the interpretation of ${ }^{18} \mathrm{~F}$ fluorodeoxyglucose Positron Emission Tomography $\left({ }^{18} \mathrm{~F}\right.$ FDG-PET) scans (Hany et al. 2002; Agrawal et al. 2009), but the potential clinical relevance of these findings was missed until the "rediscovery" of BAT. Three manuscripts published in the same issue of the New England Journal of Medicine, from the USA (Cypess et al. 2009), Finland (Virtanen et al. 2009), and the Netherlands (van Marken Lichtenbelt et al. 2009), and followed shortly by a manuscript from Japan (Saito et al. 2009), clearly indicated that BAT was present in a significant number of adults and that its presence and activity (measured by proxy as ${ }^{18} \mathrm{~F}$ FDG uptake by PET scanning) correlated with indices of healthy metabolism. Conversely, obesity, diabetes and age inversely correlated with BAT activity (Pfannenberg et al. 2010). Although the initial observations were purely correlative, the potential of a novel therapeutic target for the treatment of the metabolic consequences of obesity led to intense research to expand the capacity and activity of human BAT. Importantly, the location of adult BAT is different from the intrascapular depots observed in newborns (Drubach et al. 2011), and the transcriptome signature resembles more closely the one observed in rodent inguinal fat (Shinoda et al. 2015). This observation is relevant from the therapeutic perspective because of the exquisite plasticity of inguinal adipocytes, providing the rationale to exploit pathways to increase nonshivering thermogenesis as a means of promoting energy disposal with consequent amelioration of obesity and its metabolic consequences.

\section{Integrative Physiology Studies of Human Adaptive Thermogenesis}

To better characterize the relevance of the adaptive thermogenesis response, several integrative physiology studies were carried out in humans, mostly by exposing volunteers to cold. While a short but intense cold exposure (such as immersion of a limb in ice-cold water) is able to generate maximal ${ }^{18} \mathrm{~F}$ FDG-PET uptake and a significant increase in energy expenditure (Yoneshiro et al. 2011), this method does not correspond to day-to-day experience and cannot be sustained over time. 
Table 1 Hormonal axes and organ-system response to mild cold exposure

\begin{tabular}{l|l}
\hline Hormonal axes and organ systems & Response to cold \\
\hline Sympathetic nervous system & $\uparrow \uparrow$ \\
\hline Adrenals (medulla, epinephrine) & $\uparrow$ \\
\hline Adrenal (cortical, cortisol) & $\uparrow$ \\
\hline Thyroid axis & $\uparrow \leftrightarrow$ \\
\hline Fasting glucose & $\leftrightarrow$ \\
\hline Postprandial glucose & $\downarrow$ \\
\hline Subcutaneous adipose tissue glucose & $\leftrightarrow \downarrow$ \\
\hline Fasting insulin & $\uparrow$ \\
\hline Postprandial insulin & $\leftrightarrow$ \\
\hline Free fatty acids & $\uparrow \uparrow$ \\
\hline Subcutaneous adipose tissue lipolysis & $\leftrightarrow \uparrow$ \\
\hline FGF-21 & $\uparrow \uparrow$ \\
\hline
\end{tabular}

Additionally, intense cold exposure results in muscle fasciculation, with consequential recruitment of shivering thermogenesis. Conversely, mild cold exposure within the thermal envelope of climate-controlled buildings can stimulate a sustained physiologic adaptive thermogenesis response. In a crossover study, the adaptive thermogenesis response to minimal changes in environmental temperature was assessed in healthy volunteers who underwent two 12-h energy expenditure (EE) recordings in a whole room calorimeter (Metabolic Chamber) at 19 and $24{ }^{\circ} \mathrm{C}$ (Celi et al. 2010). The system employed in these studies recorded real-time measurements of EE and substrate utilization (respiratory quotient) while sealed ports allowed blood sampling during the study. Moreover, study volunteers were fitted with linear accelerometers and subcutaneous abdominal tissue extracellular fluid microdialysis sampling, and received a standard meal after $6 \mathrm{~h}$ of recording, enabling a comprehensive assessment of the physiologic response to short-term mild cold exposure. This complex experimental design allowed to obtain a comprehensive assessment of the physiology of adaptive thermogenesis.

The data obtained from these studies indicated that a minimal modulation of the environmental temperature was sufficient to increase EE by approximately $6 \%$, which corresponds, if projected over $24 \mathrm{~h}$, to $100 \mathrm{kcal}$ in an individual of 70-80 kg. The magnitude of this change may appear insignificant - only one fifth of the negative energy balance recommended to achieve sustained weight loss-but it is important to note that, over a 1-year period (all things being equal), these differences would be equivalent to a 20-day fast and to a daily 30-min walk at a moderate pace. Importantly, these observations also demonstrated that the adaptive thermogenesis response during mild cold exposure resulted in a significant and potentially relevant metabolic effect. A summary of the findings is reported in Table 1 (Celi et al. 2010). Exposure to mild cold is sufficient to drive an adrenergic response, which promotes lipolysis and increased postprandial glucose disposal. The significant lipolysis observed during non-shivering thermogenesis is in keeping with a functional imaging study that demonstrated that fatty acids represent the preferred substrate in BAT 
depots. On the other hand, the intervention was also sufficient to generate an increase in cortisol and a state of relative insulin resistance during fasting, which indicate an activation of the stress response.

A subsequent study correlated the individual's BAT activity, as measured by ${ }^{18} \mathrm{~F}$ FDG uptake in the thoracic region, where BAT is commonly observed with the adaptive thermogenesis response, to mild cold exposure (Chen et al. 2013). In this case, scans were acquired after a 12-h metabolic chamber recording either at 19 or $24{ }^{\circ} \mathrm{C}$ and the EE recordings were performed overnight to allow ${ }^{18} \mathrm{~F}$ FDG-PET the following morning. By comparing the tracer uptake of the two images, the overall uptake in the region of interest could be calculated and used as a linear variable to be included in multivariate statistical analyses. This strategy provided the opportunity to explore non-shivering thermogenesis as a diffuse function rather than localized in nests of brown adipocytes, overcoming the signal-to-noise ratio limitation of functional imaging. In turn, this variable was used to correlate BAT activity with metabolic and anthropometric parameters. This analysis demonstrated that BAT activity strongly correlated with the increase in EE during mild cold exposure. Remarkably, the association was present in individuals both with and without visible BAT depots on conventional ${ }^{18} \mathrm{~F}$ FDG PET imaging, indicating that diffuse (i.e., beige adipocytes-driven) uncoupling activity is an important mediator of nonshivering thermogenesis in adult humans (Chen et al. 2013).

\section{Intervention Studies Aimed to Modulate the Human Adaptive Thermogenesis Response}

These correlative studies did not address the question whether human BAT (and beige adipocytes) had the plasticity displayed by murine inguinal fat-depots, or if the capacity of BAT was sufficient to modulate a clinically significant endpoint. A short-term, moderate (10 days acclimatization with exposure at $17^{\circ} \mathrm{C}$ daily for $2 \mathrm{~h}$ ) cold exposure was sufficient to increase BAT as measured by ${ }^{18} \mathrm{~F}$ FDG-PET (van der Lans et al. 2013), whereas a similar protocol carried out for a longer period of time (6 weeks acclimatization with exposure at $17{ }^{\circ} \mathrm{C}$ daily for $2 \mathrm{~h}$ ) resulted in significant fat mass reduction (Yoneshiro et al. 2013). In a longer study, the plasticity of the adaptive thermogenesis response was assessed by modulating the environmental temperature overnight (Lee et al. 2014b). Study volunteers were exposed over a period of four consecutive months to $24^{\circ} \mathrm{C}$ (run-in period), $19{ }^{\circ} \mathrm{C}$ (cold acclimatization), $24^{\circ} \mathrm{C}$ (wash-out period), and $30^{\circ} \mathrm{C}$ (heat acclimatization). At the end of each month the adaptive thermogenesis response was studied using two consecutive metabolic chamber recordings at 19 and $24{ }^{\circ} \mathrm{C} ;{ }^{18} \mathrm{~F}$ FDG-PET for visualization and measurement of BAT activity was performed after the completion of the $19{ }^{\circ} \mathrm{C}$ metabolic chamber stay. After a month-long exposure to mild cold, BAT volume and activity nearly doubled when compared to the end of the run-in period. 
Conversely, BAT activity was negligible following a 1-month exposure to warm temperature $\left(30^{\circ} \mathrm{C}\right)$. Remarkably, the increase in BAT activity following the cold acclimatization was accompanied by a significant increase in postprandial glucose disposal, but only during mild cold exposure (Lee et al. 2014b). Thus, the data indicate that human BAT is exquisitely plastic but also that activation (i.e., cold exposure or other pharmacologic means) is necessary to generate a significant metabolic signature.

Collectively, these integrative physiology observations indicate that human BAT and beige depots represent an ideal pharmacologic target for the treatment of the consequences of a sustained positive energy balance because of their plasticity and ability to modulate carbohydrate metabolism.

\section{Manipulation of the Human Adaptive Thermogenesis Response by Hormones and Myokines}

Similarly to white adipocytes, BAT and beige adipocytes are not only targets of hormones and cytokines but also have an endocrine function, and their secretome can act in a paracrine and endocrine fashion ( $\mathrm{Ni}$ et al. 2015). Primary cultures of preadipocytes derived from the stromal vascular fraction of adipose tissues have been proven to be a robust experimental model to assess the effects of hormones and cytokines on their differentiation and function (Diaz et al. 2014). Preadipocytes can be directed toward a white or beige phenotype by hormonal manipulation of the culture media, indirectly demonstrating the plasticity of adipose tissue depots. Moreover, individual hormones, cytokines or drugs can be tested for their ability to promote "beige-ing" in white-differentiating preadipocytes. Our laboratory has worked toward defining the role of two regulators of beige adipocytes, FGF-21 and FNDC5/irisin. FGF-21 is a pleiotropic hormone that is mainly secreted by the liver and promotes insulin sensitization and browning of adipose tissue depots. Interestingly, FGF-21 is also secreted by beige adipocytes, creating an autocrine/ paracrine loop (Ni et al. 2015). In humans, FGF-21 follows a circadian rhythm that is disrupted by cold exposure (Lee et al. 2013). Compared to exposure to $24{ }^{\circ} \mathrm{C}$, exposure to mild cold resulted in an increase in the FGF-21. Importantly, the increase in FGF-21 (compared to thermoneutrality) correlated with the nonshivering thermogenesis response; this finding was also confirmed by measuring the changes in FGF-21 in relation to the lipolysis measured in the subcutaneous adipose tissue mocrodialysate (Lee et al. 2013). Collectively these observations support the role of FGF-21 in promoting and sustaining the adipocyte role in the non-shivering thermogenesis response. When FGF-21 was added to the white differentiating culture medium, human preadipocytes were directed toward a brown phenotype and were able to increase their oxygen consumption and heat production in response to norepinephrine treatment (Lee et al. 2013). These findings recapitulate the clinical 
observations that FGF-21 plays a pivotal role in beige adipocyte differentiation and function. Unfortunately, therapeutic use of FGF-21 has been limited because of concerns for off-target effects (Kharitonenkov and Adams 2014) possibly due to the systemic administration of the hormone in pharmacologic doses, unlike the local, paracrine action within the adipose tissue depots.

The discovery that BAT derives from a myf-5-positive precursor common to myocytes (Seale et al. 2008) has brought to the forefront the cross-talk between skeletal muscle and brown-beige adipocytes. Similar to adipose tissue depots, skeletal muscle has the ability to secrete myokines with hormonal activity on distant tissues. In particular, Irisin, a fibronectin-like peptide released by skeletal muscle upon contraction, has the ability to promote beige-ing in mouse inguinal adipose tissue depots and to positively affect energy and carbohydrate metabolism (Bostrom et al. 2012). The action of Irisin might appear counterintuitive since it is released by an energy-dissipating organ (skeletal muscle during contraction) and its action promotes the expansion of another energy-dissipating organ tissue (beige adipocytes). On the other hand, this apparent paradox can be reconciled once it is observed from the evolutionary perspective of energy conservation: severe cold exposure, which is able to generate shivering (highly energy inefficient) thermogenesis, promotes the expansion of the capacity of the more energy-efficient BAT and beige adipocytedriven non-shivering thermogenesis. An integrated physiology experiment was thus designed to evaluate this hypothesis by characterizing the entire spectrum of the adaptive thermogenesis response in healthy volunteers exposed to progressively lower temperatures delivered by cooling blankets (Lee et al. 2014c). This strategy allowed to capture in the same individual the vasoconstrictive response as well nonshivering and shivering thermogenesis, while the EE was annotated by indirect calorimetry (ventilated hood). Study volunteers also underwent basal and postexercise blood sampling following a maximal exercise tolerance test $\left(\mathrm{VO}_{2} \mathrm{Max}\right)$ lasting approximately $15 \mathrm{~min}$ and a 60 -min resistance exercise at $40 \% \mathrm{VO}_{2} \mathrm{Max}$. The findings of this study demonstrated a large inter-individual variability in both onset of shivering and Irisin secretion either following exercise or during controlled cooling. Furthermore, during cold exposure the release of Irisin was proportional to the shivering intensity (measured by surface electromyography). Interestingly, short-term shivering was sufficient to stimulate the release of a similar amount of Irisin when compared to maximal or resistance exercise (Lee et al. 2014c). When tested on human preadipocytes undergoing white differentiation, Irisin promoted a beige phenotype not dissimilar to the one observed following FGF-21 treatment (Lee et al. 2014a). Collectively these data confirm the role of Irisin as a myokine that is able to expand beige adipocyte mass, increasing the non-shivering thermogenesis capacity and thus promoting a shift from an inefficient (shivering) to a more efficient and sustainable form of thermogenesis (Fig. 2). 


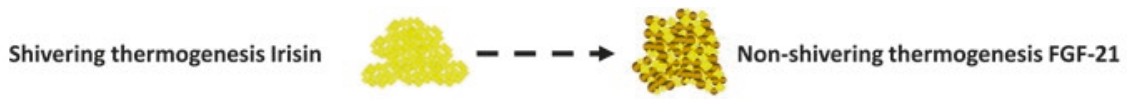

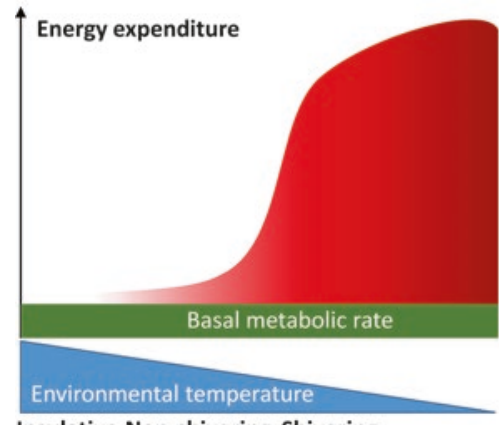

Insulative Non-shivering Shivering

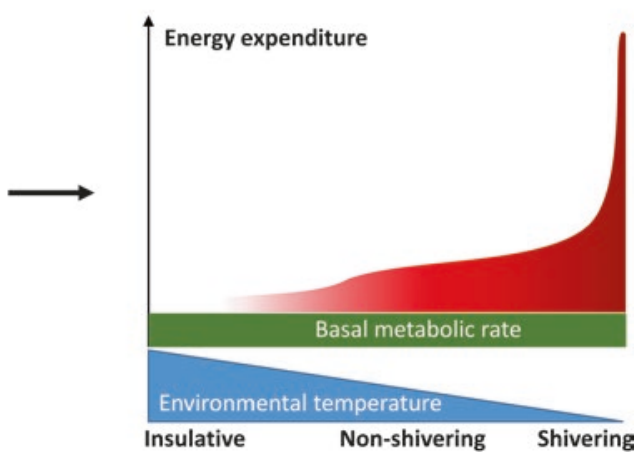

Fig. 2 Model of interplay between shivering and non-shivering thermogenesis. Exposure to cold promotes shivering in non-acclimated individuals, with release of Irisin. This response is costly from an energy-conservation perspective and not sustainable. Irisin promotes the expansion and differentiation of beige adipocytes, which increase resilience to cold enhancing non-shivering thermogenesis and delay the onset of shivering. The greater beige adipocyte mass self-sustains by the autocrine-paracrine effects of FGF-21

\section{Conclusions}

In conclusion, human integrated physiology observations coupled with translational studies offer the possibility to explore the potential therapeutic exploitation of the adaptive thermogenesis response, an evolutionary mechanism that has greatly expanded the footprint of mammalians on a wide range of climates. The ability to promote substrate utilization has the obvious appeal of an "effortless diet pill," but the capacity of the system is probably insufficient to generate alone a negative energy balance to induce weight loss. Nonetheless, even a modest increase in substrate utilization has been proven able to positively impact energy and carbohydrate metabolism. Thus, the search for novel pharmacologic interventions able to expand the beige adipocytes mass, and thereby increase the capacity for non-shivering thermogenesis, holds the promise of being a successful treatment to improve insulin resistance and the negative metabolic consequences of obesity.

\section{References}

Agrawal A, Nair N, Baghel NS (2009) A novel approach for reduction of brown fat uptake on FDG PET. Br J Radiol 82:626-631

Bostrom P, Wu J, Jedrychowski MP, Korde A, Ye L, Lo JC, Rasbach KA, Bostrom EA, Choi JH, Long JZ, Kajimura S, Zingaretti MC, Vind BF, Tu H, Cinti S, Hojlund K, Gygi SP, Spiegelman 
BM (2012) A PGC1-alpha-dependent myokine that drives brown-fat-like development of white fat and thermogenesis. Nature 481:463-468

Celi FS (2009) Brown adipose tissue — when it pays to be inefficient. N Engl J Med 360:1553-1556

Celi FS, Brychta RJ, Linderman JD, Butler PW, Alberobello AT, Smith S, Courville AB, Lai EW, Costello R, Skarulis MC, Csako G, Remaley A, Pacak K, Chen KY (2010) Minimal changes in environmental temperature result in a significant increase in energy expenditure and changes in the hormonal homeostasis in healthy adults. Eur J Endocrinol 163:863-872

Celi FS, Le TN, Ni B (2015) Physiology and relevance of human adaptive thermogenesis response. Trends Endocrinol Metab 26:238-247

Chen KY, Brychta RJ, Linderman JD, Smith S, Courville A, Dieckmann W, Herscovitch P, Millo CM, Remaley A, Lee P, Celi FS (2013) Brown fat activation mediates cold-induced thermogenesis in adult humans in response to a mild decrease in ambient temperature. J Clin Endocrinol Metab 98:E1218-E1223

Cypess AM, Lehman S, Williams G, Tal I, Rodman D, Goldfine AB, Kuo FC, Palmer EL, Tseng YH, Doria A, Kolodny GM, Kahn CR (2009) Identification and importance of brown adipose tissue in adult humans. N Engl J Med 360:1509-1517

Davis TR (1961) Chamber cold acclimatization in man. J Appl Physiol 16:1011-1015

Diaz MB, Herzig S, Vegiopoulos A (2014) Thermogenic adipocytes: from cells to physiology and medicine. Metabolism 63:1238-1249

Drubach LA, Palmer EL 3rd, Connolly LP, Baker A, Zurakowski D, Cypess AM (2011) Pediatric brown adipose tissue: detection, epidemiology, and differences from adults. J Pediatr 159:939-944

Hany TF, Gharehpapagh E, Kamel EM, Buck A, Himms-Hagen J, von Schulthess GK (2002) Brown adipose tissue: a factor to consider in symmetrical tracer uptake in the neck and upper chest region. Eur J Nucl Med Mol Imaging 29:1393-1398

Huttunen P, Hirvonen J, Kinnula V (1981) The occurrence of brown adipose tissue in outdoor workers. Eur J Appl Physiol Occup Physiol 46:339-345

Ishibashi J, Seale P (2010) Medicine. Beige can be slimming. Science 328:1113-1114

Kharitonenkov A, Adams AC (2014) Inventing new medicines: the FGF21 story. Mol Metab 3:221-229

Lee P, Brychta RJ, Linderman J, Smith S, Chen KY, Celi FS (2013) Mild cold exposure modulates fibroblast growth factor 21 (FGF21) diurnal rhythm in humans: relationship between FGF21 levels, lipolysis, and cold-induced thermogenesis. J Clin Endocrinol Metab 98:E98-102

Lee P, Werner CD, Kebebew E, Celi FS (2014a) Functional thermogenic beige adipogenesis is inducible in human neck fat. Int J Obes (Lond) 38:170-176

Lee P, Smith S, Linderman J, Courville AB, Brychta RJ, Dieckmann W, Werner CD, Chen KY, Celi FS (2014b) Temperature-acclimated brown adipose tissue modulates insulin sensitivity in humans. Diabetes 63:3686-3698

Lee P, Linderman JD, Smith S, Brychta RJ, Wang J, Idelson C, Perron RM, Werner CD, Phan GQ, Kammula US, Kebebew E, Pacak K, Chen KY, Celi FS (2014c) Irisin and FGF21 are coldinduced endocrine activators of brown fat function in humans. Cell Metab 19:302-309

Nedergaard J, Golozoubova V, Matthias A, Asadi A, Jacobsson A, Cannon B (2001) UCP1: the only protein able to mediate adaptive non-shivering thermogenesis and metabolic inefficiency. Biochim Biophys Acta 1504:82-106

Ni B, Farrar JS, Vaitkus JA, Celi FS (2015) Metabolic effects of FGF-21: thermoregulation and beyond. Front Endocrinol (Lausanne) 6:148

Petrovic N, Walden TB, Shabalina IG, Timmons JA, Cannon B, Nedergaard J (2010) Chronic peroxisome proliferator-activated receptor gamma (PPARgamma) activation of epididymally derived white adipocyte cultures reveals a population of thermogenically competent, UCP1-containing adipocytes molecularly distinct from classic brown adipocytes. J Biol Chem 285:7153-7164

Pfannenberg C, Werner MK, Ripkens S, Stef I, Deckert A, Schmadl M, Reimold M, Haring HU, Claussen CD, Stefan N (2010) Impact of age on the relationships of brown adipose tissue with sex and adiposity in humans. Diabetes 59:1789-1793 
Phillips PK, Heath JE (1995) Dependency of surface temperature regulation on body size in terrestrial mammals. J Therm Biol 20:281-289

Ravussin E, Galgani JE (2011) The implication of brown adipose tissue for humans. Annu Rev Nutr 31:33-47

Ravussin Y, Xiao C, Gavrilova O, Reitman ML (2014) Effect of intermittent cold exposure on brown fat activation, obesity, and energy homeostasis in mice. PLoS One 9:e85876

Saito M, Okamatsu-Ogura Y, Matsushita M, Watanabe K, Yoneshiro T, Nio-Kobayashi J, Iwanaga T, Miyagawa M, Kameya T, Nakada K, Kawai Y, Tsujisaki M (2009) High incidence of metabolically active brown adipose tissue in healthy adult humans: effects of cold exposure and adiposity. Diabetes 58:1526-1531

Seale P, Bjork B, Yang W, Kajimura S, Chin S, Kuang S, Scime A, Devarakonda S, Conroe HM, Erdjument-Bromage H, Tempst P, Rudnicki MA, Beier DR, Spiegelman BM (2008) PRDM16 controls a brown fat/skeletal muscle switch. Nature 454:961-967

Shinoda K, Luijten IH, Hasegawa Y, Hong H, Sonne SB, Kim M, Xue R, Chondronikola M, Cypess AM, Tseng YH, Nedergaard J, Sidossis LS, Kajimura S (2015) Genetic and functional characterization of clonally derived adult human brown adipocytes. Nat Med 21:389-394

Stocks JM, Taylor NA, Tipton MJ, Greenleaf JE (2004) Human physiological responses to cold exposure. Aviat Space Environ Med 75:444-457

van der Lans AA, Hoeks J, Brans B, Vijgen GH, Visser MG, Vosselman MJ, Hansen J, Jorgensen JA, Wu J, Mottaghy FM, Schrauwen P, van Marken Lichtenbelt WD (2013) Cold acclimation recruits human brown fat and increases nonshivering thermogenesis. J Clin Invest 123:3395-3403

van Marken Lichtenbelt WD, Vanhommerig JW, Smulders NM, Drossaerts JM, Kemerink GJ, Bouvy ND, Schrauwen P, Teule GJ (2009) Cold-activated brown adipose tissue in healthy men. N Engl J Med 360:1500-1508

Vianna CR, Hagen T, Zhang CY, Bachman E, Boss O, Gereben B, Moriscot AS, Lowell BB, Bicudo JE, Bianco AC (2001) Cloning and functional characterization of an uncoupling protein homolog in hummingbirds. Physiol Genomics 5:137-145

Virtanen KA, Lidell ME, Orava J, Heglind M, Westergren R, Niemi T, Taittonen M, Laine J, Savisto NJ, Enerback S, Nuutila P (2009) Functional brown adipose tissue in healthy adults. N Engl J Med 360:1518-1525

Yoneshiro T, Aita S, Matsushita M, Kameya T, Nakada K, Kawai Y, Saito M (2011) Brown adipose tissue, whole-body energy expenditure, and thermogenesis in healthy adult men. Obesity (Silver Spring) 19:13-16

Yoneshiro T, Aita S, Matsushita M, Kayahara T, Kameya T, Kawai Y, Iwanaga T, Saito M (2013) Recruited brown adipose tissue as an antiobesity agent in humans. J Clin Invest 123:3404-3408

Open Access This chapter is licensed under the terms of the Creative Commons Attribution 4.0 International License (http://creativecommons.org/licenses/by/4.0/), which permits use, sharing, adaptation, distribution and reproduction in any medium or format, as long as you give appropriate credit to the original author(s) and the source, provide a link to the Creative Commons license and indicate if changes were made.

The images or other third party material in this chapter are included in the chapter's Creative Commons license, unless indicated otherwise in a credit line to the material. If material is not included in the chapter's Creative Commons license and your intended use is not permitted by statutory regulation or exceeds the permitted use, you will need to obtain permission directly from the copyright holder.

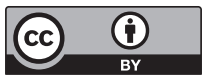

